\title{
Reversible Logic-Based Fault-Tolerant Nanocircuits in QCA
}

\author{
Bibhash Sen, ${ }^{1}$ Siddhant Ganeriwal, ${ }^{1}$ and Biplab K. Sikdar ${ }^{2}$ \\ ${ }^{1}$ Department of Computer Science and Engineering, National Institute of Technology, Durgapur 713209, India \\ ${ }^{2}$ Department of Computer Science and Technology, Bengal Engineering and Science University, Shibpur 711103, India
}

Correspondence should be addressed to Bibhash Sen; bibhash.sen@gmail.com

Received 28 April 2013; Accepted 26 May 2013

Academic Editors: D. Rossi and P. Wachulak

Copyright (C) 2013 Bibhash Sen et al. This is an open access article distributed under the Creative Commons Attribution License, which permits unrestricted use, distribution, and reproduction in any medium, provided the original work is properly cited.

\begin{abstract}
Parity-preserving reversible circuits are gaining importance for the development of fault-tolerant systems in nanotechnology. On the other hand, Quantum-dot Cellular Automata (QCA), a potential alternative to CMOS, promises efficient digital design at nanoscale. This work targets design of reversible ALU (arithmetic logic unit) in QCA (Quantum-dot Cellular Automata) framework. The design is based on the fault tolerant reversible adders (FTRA) introduced in this paper. The proposed fault tolerant adder is a parity-preserving gate, and QCA implementation of FTRA achieved $47.38 \%$ fault-free output in the presence of all possible single missing/additional cell defects. The proposed designs are verified and evaluated over the existing ALU designs and found to be more efficient in terms of design complexity and quantum cost.
\end{abstract}

\section{Introduction}

Reversible logic has attractive perspective of constructing digital devices that can realize computing unit with almost zero power dissipation. Landauer [1] proved that for irreversible computations, each bit of information loss generates $k_{B} T \ln 2$ joules of heat energy. The energy $E_{\mathrm{bit}}$ required for a binary transition is given by SNL (Shannon-Von NeumannLandauer) expression in [1] as follows:

$$
E_{\mathrm{bit}} \geq E_{\mathrm{SNL}}=k_{B} T \ln 2=0.017 \mathrm{eV},
$$

where $k_{B}$ is Boltzmann constant and $T=300 \mathrm{~K}$. This is the minimum energy to process a bit. Bennett [2] showed that a zero power dissipation in logic circuit is possible only if the circuit is composed of reversible logic gates. Since QCA circuits are clocked information preserving systems, the energy dissipation of QCA circuits can be significantly lower than $k_{B} T \ln 2$. This feature favours the introduction of QCA technology in reversible logic design.

Though, reversibility recovers bit loss, but it is not able to detect bit error in circuit. Fault-tolerant reversible circuits are capable of preventing errors at outputs. If the system itself made of fault-tolerant components, then the detection and correction of faults become easier and simple. In communication and many other systems, fault tolerance is achieved by parity. Therefore, parity-preserving reversible circuits will be the future design trends to the development of fault-tolerant reversible systems in nanotechnology.

On the other hand, QCA (Quantum-dot Cellular Automata) is considered to be promising in the field of nanotechnology due to their extremely small sizes and ultralow-power consumption [3]. The QCA is based on encoding binary information in the charge configuration of quantum-dot cells. The interaction between cells is coulombic and provides the necessary computing power. The fundamental unit of QCAbased design is the 3-input majority gate [3]. Majority gate with inverter, called MI, is used to realize the QCA designs because of its functional incompleteness.

Significant contributions have been made in the literature towards the design of arithmetic units in [4-6]. Also, faulttolerant full adder circuits are explored in [7-10]. However, there have only been a few efforts towards designing reversible fault-tolerant ALUs.

This motivates us to design a fault-tolerant reversible ALU architecture considering QCA technology. A cost-effective realization of a fault-tolerant reversible adder (FTRA) is first introduced. It is then utilized to synthesize the desired faulttolerant reversible arithmetic logic unit which outperforms the efficiency of existing designs in terms of design complexity and quantum cost. The major contributions of this work 
around reversible QCA architecture can be summarized as follows:

(i) realization of generic fault-tolerant reversible adder having parity-preserving logic with cost effective quantum cost;

(ii) the presented fault-tolerant reversible adder block is used to realize different arithmetic circuit such as full adder, subtractor, ripple carry adder, and carry-skip logic;

(iii) synthesis of a fault-tolerant reversible arithmetic logic unit (ALU) using proposed adder;

(iv) application of the proposed adder in QCA nanotechnology with effective fault tolerance of $47.38 \%$ in the presence of all possible single missing/additional cell defects.

Simulations using QCADesigner [11] supports all the results presented.

\section{Preliminaries}

Reversible Logic. A logic gate is reversible if the mapping of its inputs to outputs is bijective; that is, every distinct input yields a distinct output, and the number of inputs is equal to the number of outputs [12]. An important cost metrics in reversible logic circuits is the quantum cost. The cost of every $2 \times 2$ gate is the same; that is, unity and the cost of $1 \times 1$ gate are zero [13]. Any reversible logic can be realized using primitive quantum gates, like $1 \times 1$ NOT gates and $2 \times 2$ reversible gates, such as Controlled- $V$, Controlled $-V^{+}$, and CNOT gate (Figure 1). The quantum cost of a reversible gate can be calculated by counting the numbers of primitive quantum gates used in implementing it. The fan-outs and feedback paths are not permitted in reversible logic.

QCA Basics. A QCA cell consists of four quantum dots positioned at the corners of a square (Figure 2(a)) and contains two free electrons [3]. The electrons can quantum mechanically tunnel among the dots and settle either in polarization $\mathrm{P}=-1(\operatorname{logic} 0)$ or in $\mathrm{P}=+1(\operatorname{logic} 1)$ as shown in Figure 2(b). Timing in QCA is accomplished by the cascaded clocking of four distinct and periodic phases [3]. The basic structure realized with QCA is the 3-input majority gate, $\operatorname{MV}(A, B, C)=\operatorname{Maj}(A, B, C)=A B+B C+C A($ Figure 2(c)). It can also function as a 2-input AND (2-input $\mathrm{OR}$ ) logic by fixing one of the three input cells to $\mathrm{P}=-1(\mathrm{P}=+1)$. Inverter is realized in two different orientations as shown in Figure 2(d). In QCA-based logic implementations, two kinds of wire crossover, termed coplanar crossover and multilayer crossover, are possible. Figure 2(e) describes the coplaner wire crossing considering a $90^{\circ}\left(\mathrm{x}\right.$-cell) and a $45^{\circ}(+$-cell $)$ structures.

Fault-Tolerant Logic. Fault tolerance enables a system to operate properly in the event of the failure of some its components. If the system itself is made up of fault-tolerant components, then the detection and correction of faults become easier and simple. A fault-tolerant (FT) reversible gate is also called
TABLE 1: Truth table of proposed fault-tolerant adder.

\begin{tabular}{cccccccccc}
\hline $\mathrm{A}$ & $\mathrm{B}$ & $\mathrm{C}$ & $\mathrm{D}$ & $\mathrm{E}$ & $\mathrm{P}$ & $\mathrm{Q}$ & $\mathrm{R}$ & $\mathrm{S}$ & $\mathrm{T}$ \\
\hline 0 & 0 & 0 & 0 & 0 & 0 & 0 & 0 & 0 & 0 \\
0 & 0 & 0 & 0 & 1 & 0 & 0 & 0 & 0 & 1 \\
0 & 0 & 0 & 1 & 0 & 0 & 0 & 1 & 1 & 1 \\
0 & 0 & 0 & 1 & 1 & 0 & 0 & 1 & 1 & 0 \\
0 & 0 & 1 & 0 & 0 & 0 & 0 & 1 & 0 & 0 \\
0 & 0 & 1 & 0 & 1 & 0 & 0 & 1 & 0 & 1 \\
0 & 0 & 1 & 1 & 0 & 0 & 0 & 0 & 1 & 1 \\
0 & 0 & 1 & 1 & 1 & 0 & 0 & 0 & 1 & 0 \\
0 & 1 & 0 & 0 & 0 & 0 & 1 & 1 & 0 & 1 \\
0 & 1 & 0 & 0 & 1 & 0 & 1 & 1 & 0 & 0 \\
0 & 1 & 0 & 1 & 0 & 0 & 1 & 0 & 0 & 1 \\
0 & 1 & 0 & 1 & 1 & 0 & 1 & 0 & 0 & 0 \\
0 & 1 & 1 & 0 & 0 & 0 & 1 & 0 & 1 & 0 \\
0 & 1 & 1 & 0 & 1 & 0 & 1 & 0 & 1 & 1 \\
0 & 1 & 1 & 1 & 0 & 0 & 1 & 1 & 1 & 0 \\
0 & 1 & 1 & 1 & 1 & 0 & 1 & 1 & 1 & 1 \\
1 & 0 & 0 & 0 & 0 & 1 & 0 & 1 & 0 & 0 \\
1 & 0 & 0 & 0 & 1 & 1 & 0 & 1 & 0 & 1 \\
1 & 0 & 0 & 1 & 0 & 1 & 0 & 0 & 0 & 0 \\
1 & 0 & 0 & 1 & 1 & 1 & 0 & 0 & 0 & 1 \\
1 & 0 & 1 & 0 & 0 & 1 & 0 & 0 & 1 & 1 \\
1 & 0 & 1 & 0 & 1 & 1 & 0 & 0 & 1 & 0 \\
1 & 0 & 1 & 1 & 0 & 1 & 0 & 1 & 1 & 1 \\
1 & 0 & 1 & 1 & 1 & 1 & 0 & 1 & 1 & 0 \\
1 & 1 & 0 & 0 & 0 & 1 & 1 & 0 & 1 & 0 \\
1 & 1 & 0 & 0 & 1 & 1 & 1 & 0 & 1 & 1 \\
1 & 1 & 0 & 1 & 0 & 1 & 1 & 1 & 0 & 1 \\
1 & 1 & 0 & 1 & 1 & 1 & 1 & 1 & 0 & 0 \\
1 & 1 & 1 & 0 & 0 & 1 & 1 & 1 & 1 & 0 \\
1 & 1 & 1 & 0 & 1 & 1 & 1 & 1 & 1 & 1 \\
1 & 1 & 1 & 1 & 0 & 1 & 1 & 0 & 0 & 1 \\
1 & 1 & 1 & 1 & 1 & 1 & 1 & 0 & 0 & 0 \\
\hline & & & & & & & & &
\end{tabular}

conservative gate [7]. The hamming weight of its inputs and outputs is equal. Let the input and output vectors of any faulttolerant gate be $I_{v}=I_{0}, I_{1}, \ldots, I_{n-1}$ and $O_{v}=O_{0}, O_{1}, \ldots, O_{n-1}$, where
(i) $I_{v}\langle$ Bijective $\rangle O_{v}$,
(ii) $I_{0} \oplus I_{1} \oplus \cdots \oplus I_{n-1}=O_{0} \oplus O_{1} \oplus \cdots \oplus O_{n-1}$.

Few fault-tolerant reversible $3 \times 3$ gate-like Feynman double gate (F2G), Fredkin (FRG), NFT, and so forth and $4 \times 4$ gate like MIG are already investigated.

\section{Related Work}

In [14], the feasibility of the parity-preserving approach to design of reversible logic circuits was explored. Few new fault-tolerant reversible logic gates were proposed in [8]. Fault-tolerant full adder circuits are explored in [7-10]. In all these researches, no such single logic unit was identified to implement fault-tolerant full adder. Also, the fault tolerance 


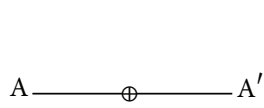

(a)

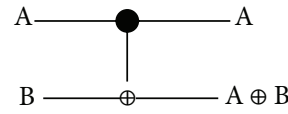

(b)

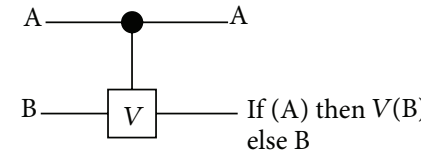

(c)

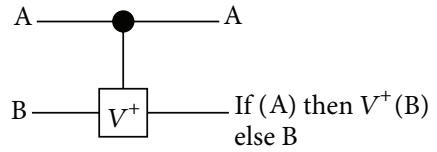

(d)

FIgURE 1: Elementary quantum logic gates: (a) NOT, (b) exclusive OR, (c) square root of NOT (SRN), and (d) Hermitian matrix of SRN.

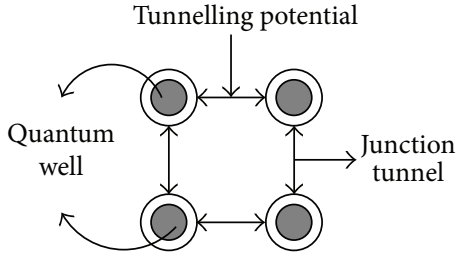

(a) Structure of a QCA Cell

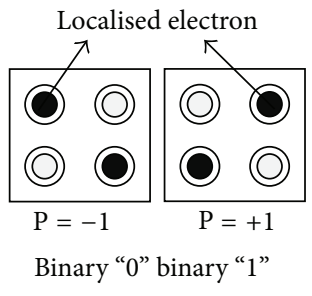

(b) QCA cell with two different polarizations

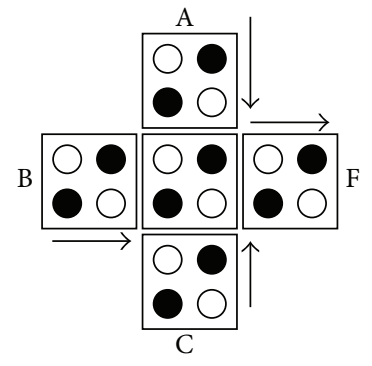

(c) Majority voter

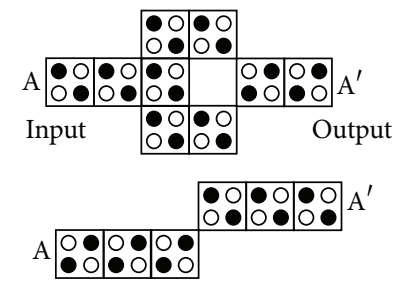

(d) Inverter

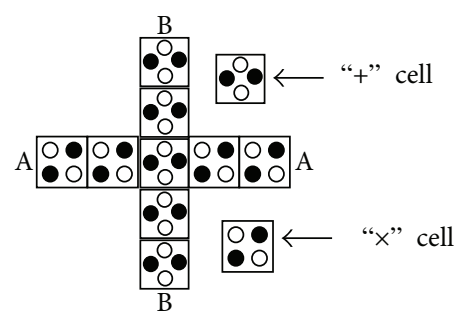

(e) Wire crossing

FIGURE 2: QCA basics.

capability of those logic gates was not explored quantitatively. Significant contributions have been made in the literature towards the design of arithmetic units in [4-6]. However, there have only been a few efforts towards designing reversible fault-tolerant ALUs. A new fault-tolerant method based on majority multiplexing (Maj-MUX) is proposed for QCA in [15]. But the application of reversible logic in QCA to achieve fault tolerance was not identified (which is of primary interest to us in this work).

\section{Proposed Fault-Tolerant Adder}

In communication and many other systems, fault tolerance is achieved by parity. A parity-preserving reversible gate, when used with an arbitrary synthesis strategy for reversible logic circuits, allows any fault that affects no more than a single logic signal to be detectable at the circuit's primary outputs [14]. In [10], a minimum number of garbage outputs and constant inputs for a fault-tolerant reversible full adder circuit are specified as 3 and 2. Keeping in mind to have minimum number of garbage outputs and constant inputs, a new fault-tolerant reversible adder (FTRA) is proposed here (Figure 3(a)) followed by design of different adder-based circuits. The input to output mapping of FTRA structure is $\mathrm{P}=\mathrm{A}, \mathrm{Q}=\mathrm{A} \oplus \mathrm{B}, \mathrm{R}=\mathrm{A} \oplus \mathrm{B} \oplus \mathrm{C} \oplus \mathrm{D}, \mathrm{S}=(\mathrm{A} \oplus \mathrm{B})(\mathrm{C} \oplus \mathrm{D})(\mathrm{AB} \oplus \mathrm{D})$, and $\mathrm{T}=(\mathrm{A} \oplus \mathrm{B})(\mathrm{C} \oplus \mathrm{D})(\overline{\mathrm{A}} \mathrm{B} \oplus \mathrm{D}) \oplus \mathrm{E}$, where $\mathrm{A}, \mathrm{B}, \mathrm{C}, \mathrm{D}$, and $\mathrm{E}$ are inputs and $\mathrm{P}, \mathrm{Q}, \mathrm{R}, \mathrm{S}$, and $\mathrm{T}$ are outputs. It is a $5 \times 5$ reversible gate with a quantum cost of 8 (Figure $3(\mathrm{~b})$ ). The proposed reversible FTRA gate is parity preserving. The reversibility and parity preserve nature of the proposed FTRA gate can be verified from Table 1 .

A fault-tolerant reversible full adder and subtractor circuit using the newly proposed FTRA gate is shown in Figure 4(a). The design uses only one FTRA gate, has a quantum cost of 8 , produces 3 garbage outputs, and uses 2 constant inputs only. No such single logic gate is found which can implement a fault-tolerant reversible full adder with such quantum cost and less complexity. Design capability of the proposed adder is further analyzed by implementing a 4bit fault-tolerant ripple carry adder (Figure 4(b)) and carry skip adder (Figure 4(c)). A comparative performance analysis of the proposed design with existing designs is reported in Table 2 with enviable gate count besides other parameters. The results shows that the proposed design is much more effective than the existing designs.

The advantage of our method is in the implementation of this logic at gate level. Thus, once the required gates have been designed and an appropriate synthesis framework has been established, fault-tolerant implementation requires no extra expenditure in design or verification effort. 


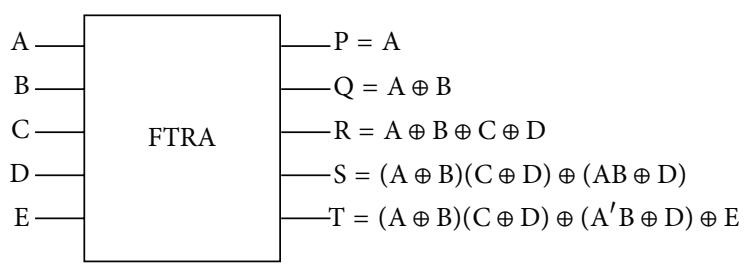

(a) Block diagram fault-tolerant adder

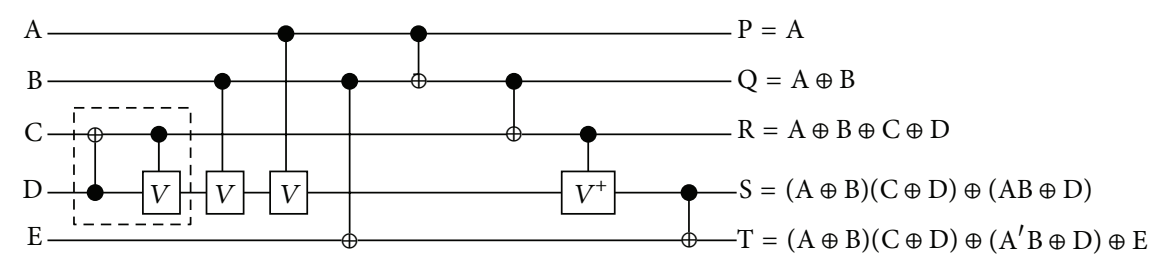

(b) Quantum implementation of fault-tolerant adder

FIgURE 3: Proposed fault-tolerant reversible adder.

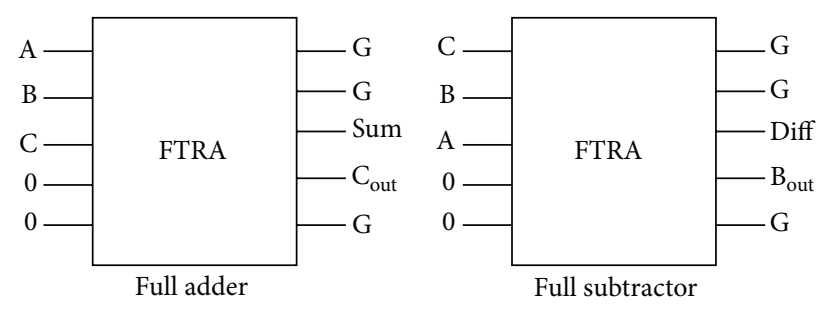

(a) Fault-tolerant full adder and subtractor

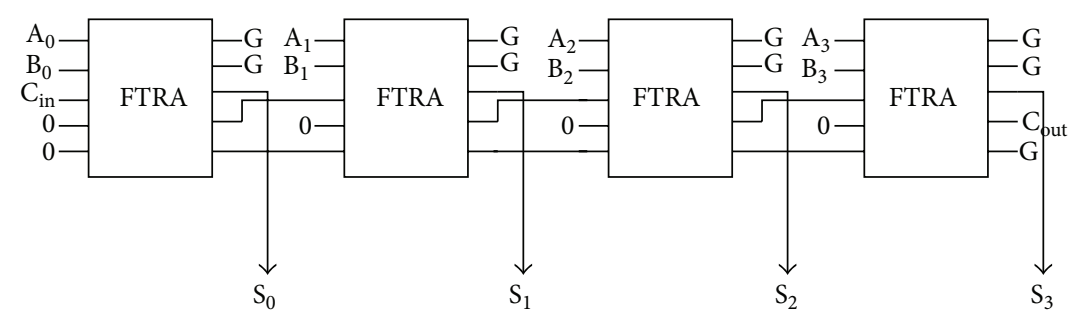

(b) Fault-tolerant ripple carry adder

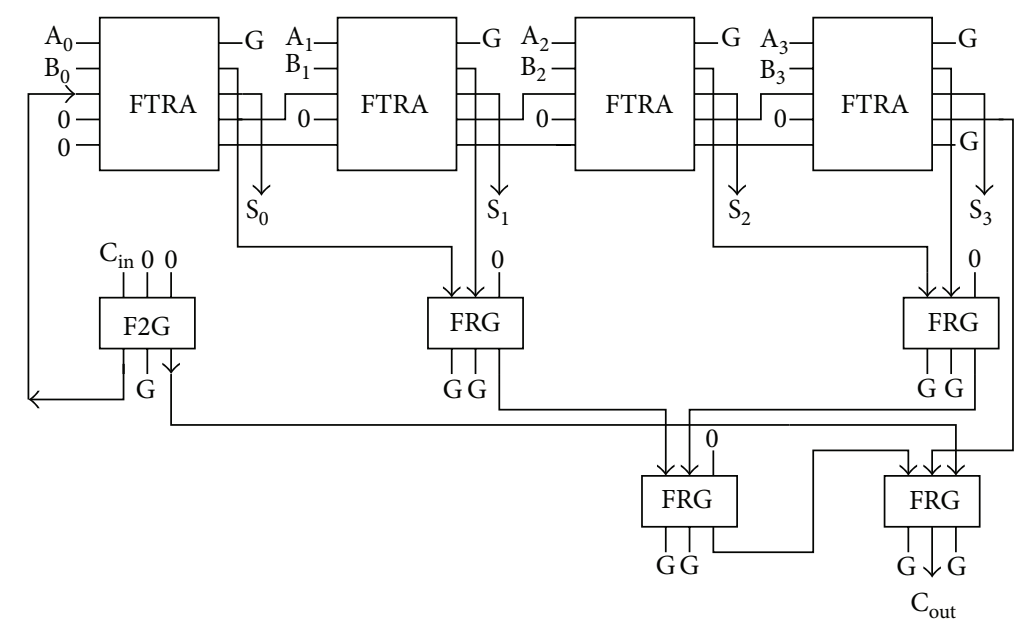

(c) Fault-tolerant carry-skip adder

FIGURE 4: Implementation of different logic circuit with proposed FTRA. 


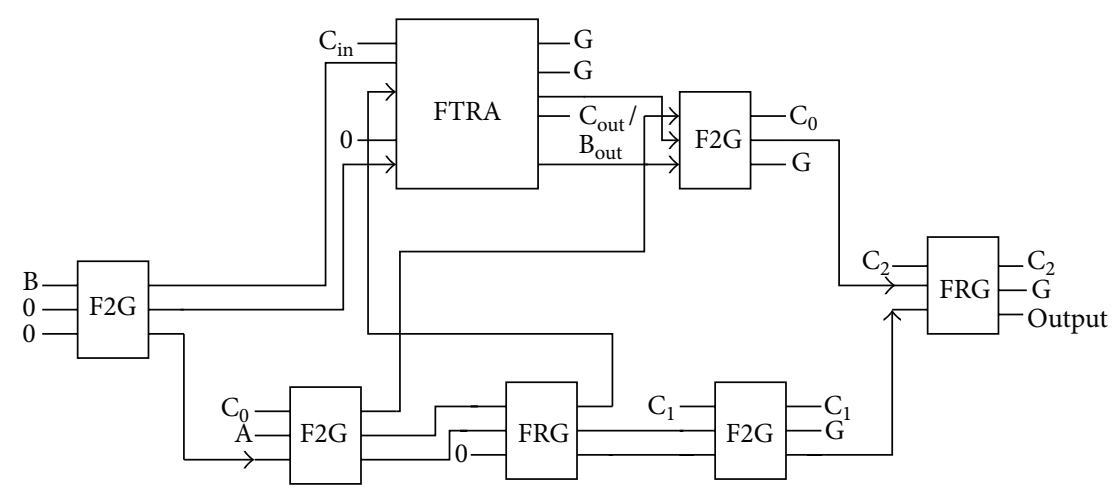

FIgURE 5: Fault-tolerant ALU with proposed FTRA.

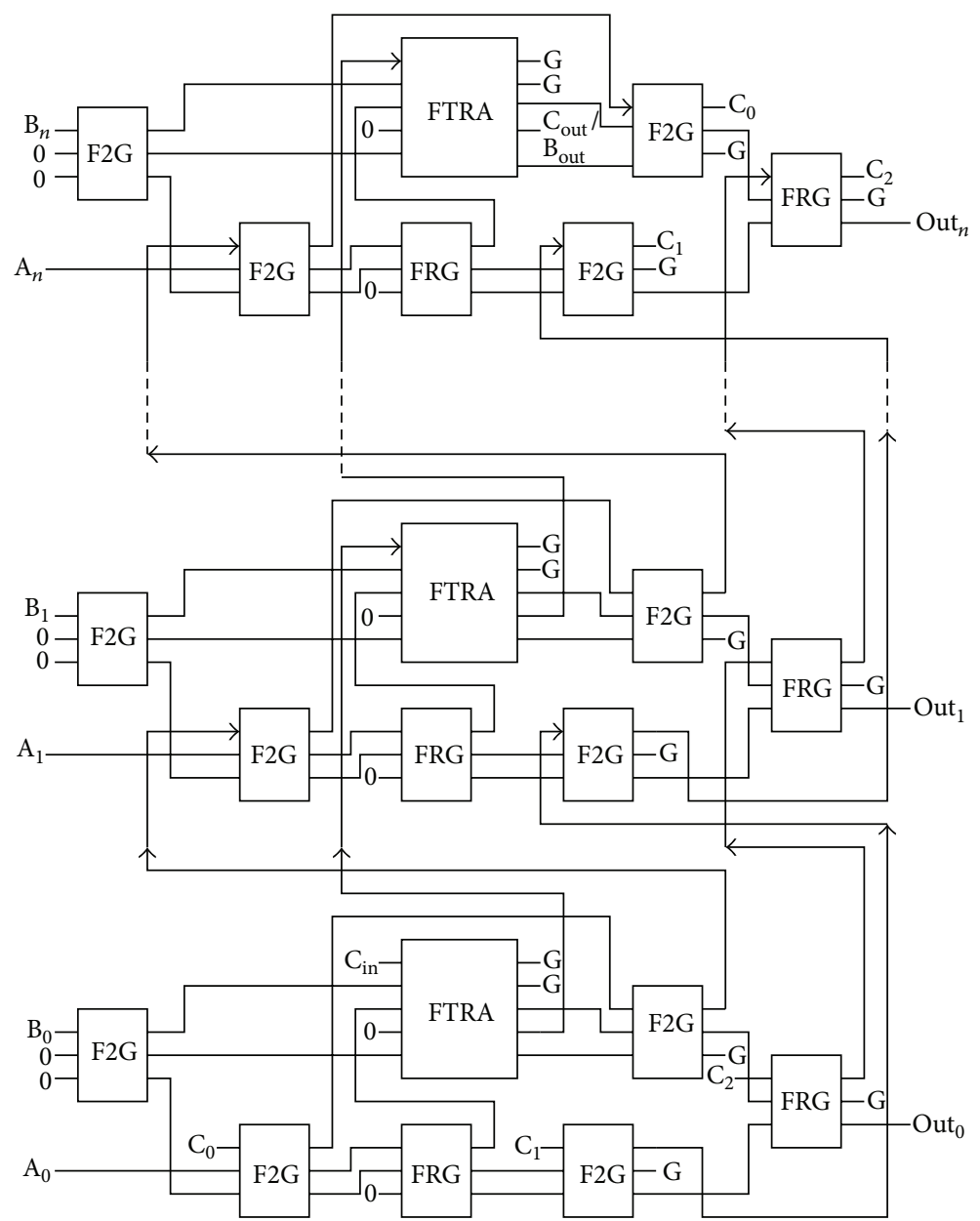

FIGURE 6: Implementation of n-bit fault-tolerant ALU.

TABLE 2: Performance analysis of different fault-tolerant full adders.

\begin{tabular}{|c|c|c|c|c|c|}
\hline Parameter & [7] & [8] & [9] & {$[10]$} & Proposed \\
\hline Gate count & 5 & 6 & 2 & 4 & 1 \\
\hline Quantum cost & 25 & 18 & 14 & 11 & 8 \\
\hline Garbage outputs & 4 & 6 & 3 & 3 & 3 \\
\hline Constant inputs & 2 & 5 & 2 & 2 & 2 \\
\hline Logical calculations & $8 \alpha+16 \beta+8 \delta$ & $12 \alpha+8 \beta+4 \delta$ & $8 \alpha+6 \beta+2 \delta$ & $9 \alpha+4 \beta+3 \delta$ & $13 \alpha+4 \beta+\delta$ \\
\hline
\end{tabular}

$\alpha$ : "two-input EXOR calculation"; $\beta$ : "two-input AND calculation"; $\delta$ : "NOT calculation." 


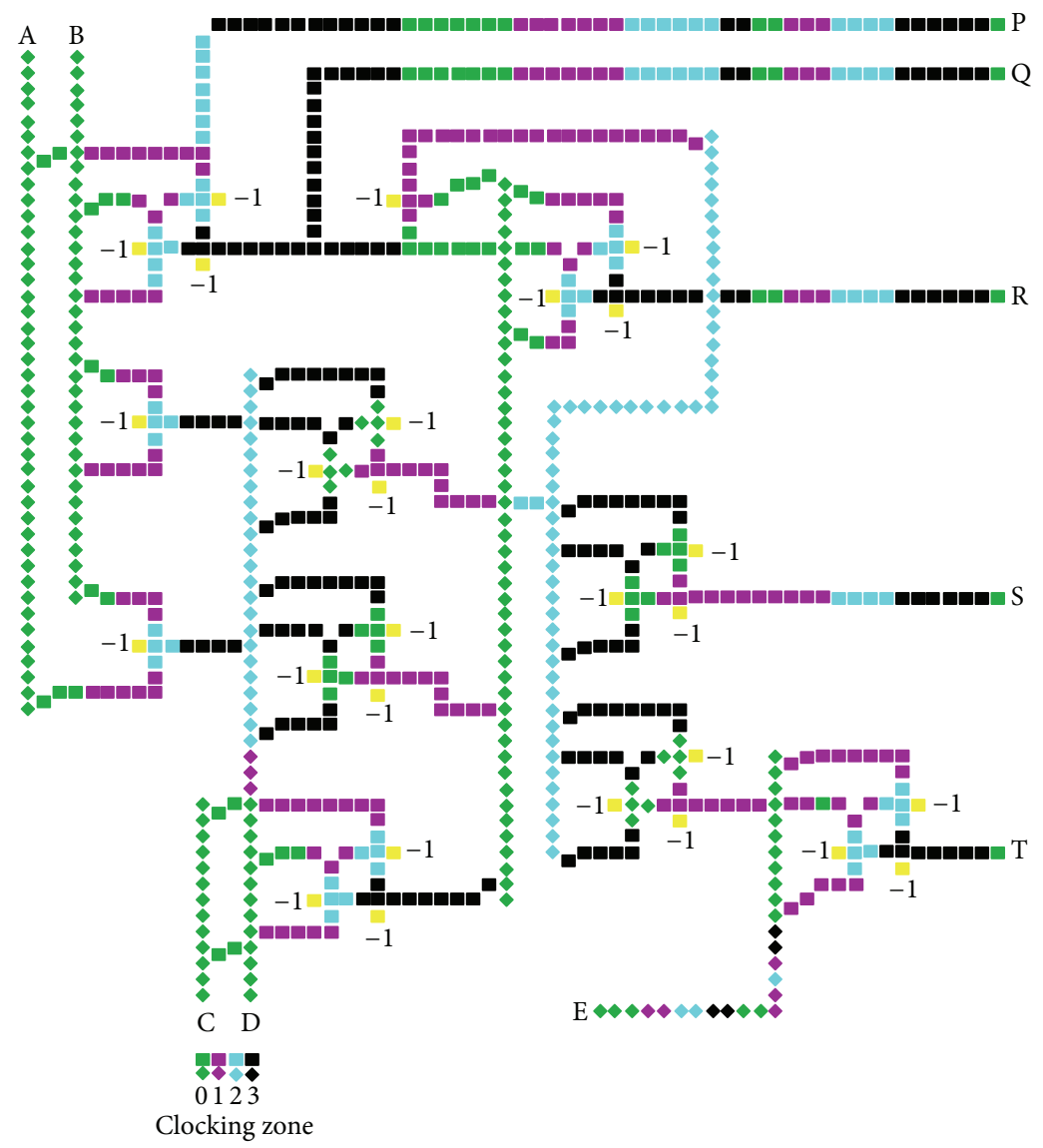

FIGURE 7: QCA implementation of fault-tolerant adder.

TABLE 3: Different function of ALU.

\begin{tabular}{lcccc}
\hline $\mathrm{C} 0$ & $\mathrm{C} 1$ & $\mathrm{C} 2$ & Output & Function \\
\hline 0 & 0 & 0 & $\mathrm{AB}$ & $\mathrm{AND}$ \\
0 & 0 & 1 & $\overline{\mathrm{A}+\mathrm{B}}$ & $\mathrm{NOR}$ \\
0 & 1 & 0 & $\overline{\mathrm{AB}}$ & NAND \\
0 & 1 & 1 & $\mathrm{~A}+\mathrm{B}$ & $\mathrm{OR}$ \\
1 & $\mathrm{X}$ & 0 & $\mathrm{~A} \oplus \mathrm{B} \oplus \mathrm{C}$ & $\mathrm{ADD}$ \\
1 & $\mathrm{X}$ & 1 & $\mathrm{~A} \oplus \mathrm{B} \oplus \mathrm{C}$ & $\mathrm{SUB}$ \\
\hline
\end{tabular}

4.1. Design of Fault-Tolerant Arithmetic Logic Unit (ALU). The reversible fault-tolerant 1-bit ALU is designed with one FTRA gate, two Fredkin gates, and four Double-Feynman gates. Thus the design uses a total of 7 gates and has a quantum cost of 26 (Figure 5). It produces 5 garbage outputs and uses 4 constant inputs. The various operations performed by the proposed FTRA-ALU is shown in Table 3. The total logical operations performed is $21 \alpha+10 \beta+5 \delta$. The main advantage of the proposed ALU logic is its programmable feature; that is, just programming the constants $\mathrm{C} 1, \mathrm{C} 2$, and $\mathrm{C} 3$, different ALU functions are implemented. Besides its fault tolerance, this programmable feature adds more flexibility in reversible ALU. Figure 6 shows the design of an n-bit fault-tolerant ALU which is synthesized by cascading the 1-bit ALU module explored in Figure 5.

\section{FTRA Gates in QCA Computing}

To demonstrate the application of the proposed fault-tolerant design approach to reversible adder in emerging nano-technologies, Quantum-dot cellular automata (QCA) technology is considered because reversible logic has potential applications in QCA computing. The QCA implementation of the proposed FTRA gate is shown in Figure 7. The design requires $27 \mathrm{MVs}$ and has a delay of 12 clock zones. In the following section, the fault tolerance capability is established quantitatively. FTRA simulation shown in Figure 8 is performed by fixing $\mathrm{D}$ and $\mathrm{E}$ to 0 . In this simulation result, $\mathrm{R}$ generates sum, and $\mathrm{S}$ propagates carry.

\section{Fault Tolerance of Proposed Logic Gate}

In QCA manufacturing, defects can occur during the synthesis and deposition phases, although defects are most likely to take place during the deposition phase [16]. Researchers have shown that QCA cells are more susceptible to missing and additional QCA cell defects [17]. In additional cell defect, an additional cell is deposited on the substrate. The missing cell defect occurs due to the missing of a particular cell. Researchers have been addressing the design and test of QCA circuits assuming the single missing/additional cell defect model. 


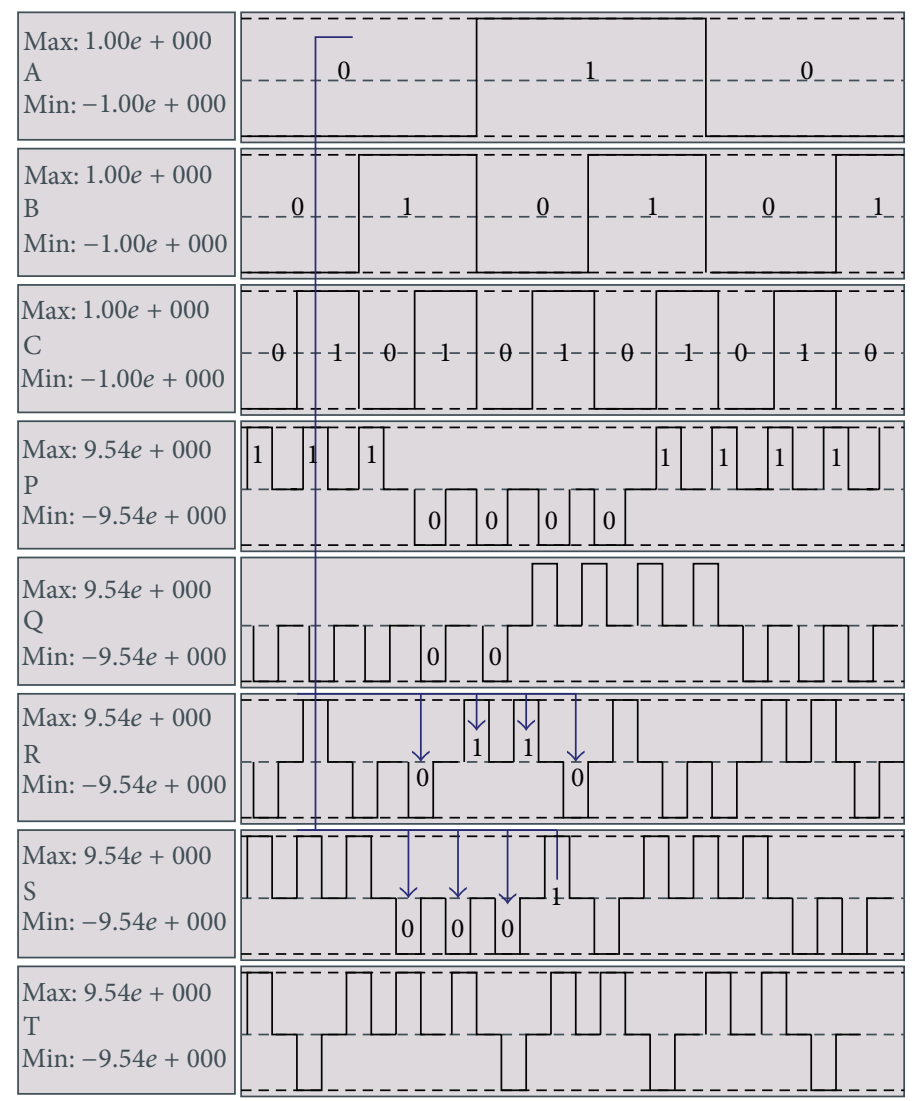

FIGURE 8: Simulation result of fault-tolerant QCA adder.

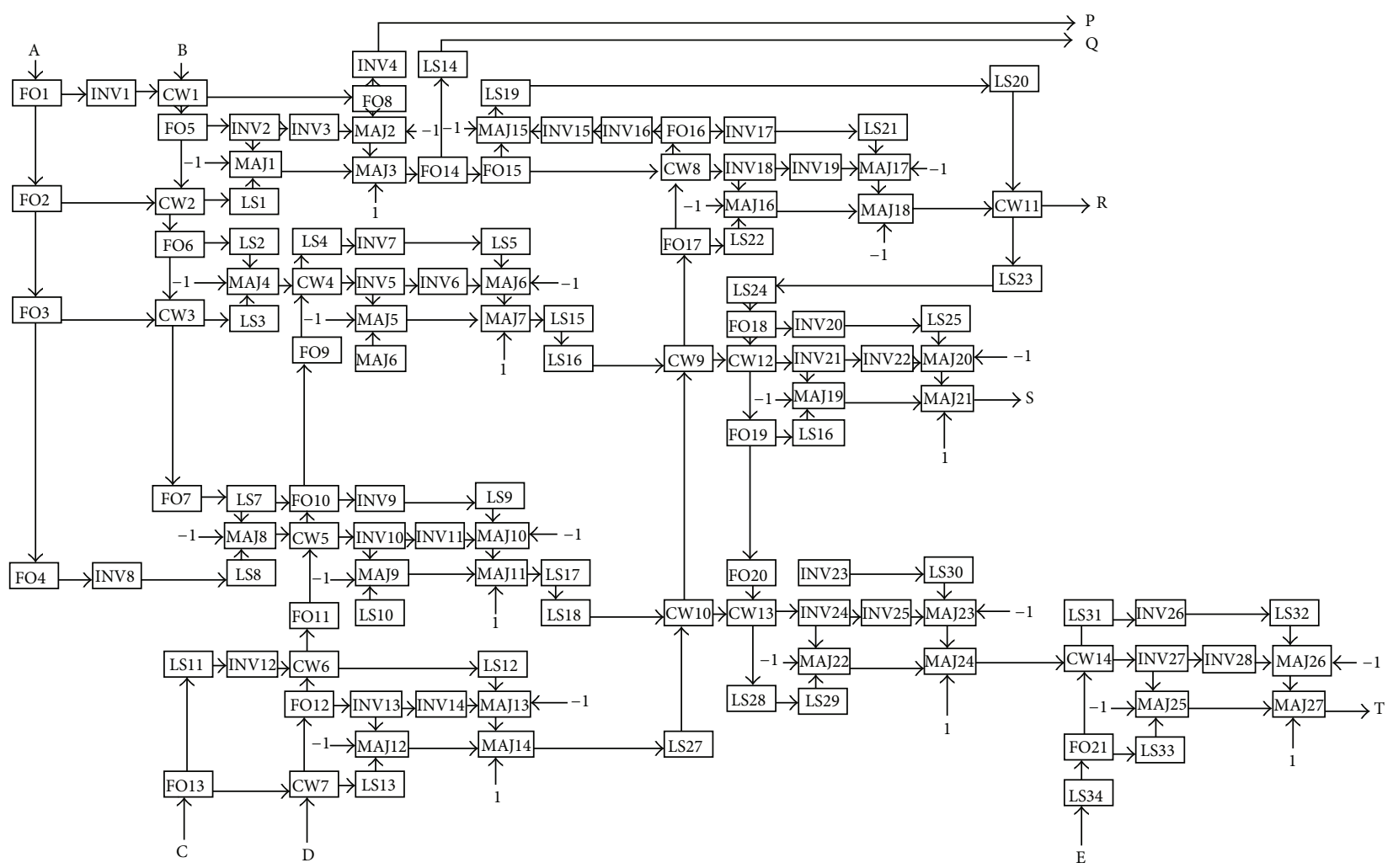

Figure 9: HDLQ modelling of QCA-based fault-tolerant adder. 
TABLE 4: Fault pattern of proposed fault-tolerant adder.

\begin{tabular}{|c|c|c|c|c|c|c|c|c|c|c|c|c|c|c|}
\hline \multirow{2}{*}{ Input } & \multirow{2}{*}{ Output } & \multicolumn{11}{|c|}{ Fault pattern } & \multirow{2}{*}{ Success rate } & \multirow{2}{*}{ FT\% } \\
\hline & & 1 & 2 & 3 & $\ldots$ & 43 & 44 & 45 & $\ldots$ & 76 & 77 & 78 & & \\
\hline$a_{0}$ & $a_{0}$ & $a_{16}$ & $a_{12}$ & $a_{0}$ & $\cdots$ & $a_{2}$ & $a_{1}$ & $a_{0}$ & $\cdots$ & $a_{12}$ & $a_{1}$ & $a_{0}$ & $32 / 78$ & 41.02 \\
\hline$a_{1}$ & $a_{1}$ & $a_{17}$ & $a_{13}$ & $a_{1}$ & $\cdots$ & $a_{3}$ & $a_{0}$ & $a_{1}$ & $\cdots$ & $a_{1}$ & $a_{0}$ & $a_{0}$ & $34 / 78$ & 43.59 \\
\hline$a_{2}$ & $a_{7}$ & $a_{23}$ & $a_{8}$ & $a_{7}$ & $\cdots$ & $a_{5}$ & $a_{6}$ & $a_{7}$ & $\cdots$ & $a_{7}$ & $a_{6}$ & $a_{7}$ & $38 / 78$ & 48.72 \\
\hline$a_{3}$ & $a_{6}$ & $a_{22}$ & $a_{8}$ & $a_{7}$ & $\cdots$ & $a_{4}$ & $a_{7}$ & $a_{6}$ & $\cdots$ & $a_{6}$ & $a_{6}$ & $a_{6}$ & $38 / 78$ & 48.72 \\
\hline$a_{4}$ & $a_{4}$ & $a_{20}$ & $a_{11}$ & $a_{4}$ & $\cdots$ & $a_{6}$ & $a_{5}$ & $a_{4}$ & $\cdots$ & $a_{5}$ & $a_{4}$ & $a_{4}$ & $37 / 78$ & 47.43 \\
\hline$a_{5}$ & $a_{5}$ & $a_{21}$ & $a_{10}$ & $a_{5}$ & $\cdots$ & $a_{7}$ & $a_{4}$ & $a_{5}$ & $\cdots$ & $a_{5}$ & $a_{5}$ & $a_{5}$ & $39 / 78$ & 50.00 \\
\hline$a_{6}$ & $a_{3}$ & $a_{19}$ & $a_{15}$ & $a_{3}$ & $\cdots$ & $a_{1}$ & $a_{2}$ & $a_{3}$ & $\cdots$ & $a_{3}$ & $a_{2}$ & $a_{3}$ & $40 / 78$ & 51.28 \\
\hline$\vdots$ & $\vdots$ & $\vdots$ & : & $\vdots$ & $\vdots$ & $\vdots$ & $\vdots$ & $\vdots$ & $\vdots$ & $\vdots$ & $\vdots$ & $\vdots$ & $\vdots$ & $\vdots$ \\
\hline$a_{30}$ & $a_{17}$ & $a_{13}$ & $a_{17}$ & $a_{19}$ & $\cdots$ & $a_{19}$ & $a_{16}$ & $a_{17}$ & $\cdots$ & $a_{17}$ & $a_{16}$ & $a_{17}$ & $41 / 78$ & 52.56 \\
\hline$a_{31}$ & $a_{16}$ & $a_{12}$ & $a_{16}$ & $a_{18}$ & $\cdots$ & $a_{18}$ & $a_{17}$ & $a_{16}$ & $\cdots$ & $a_{16}$ & $a_{16}$ & $a_{16}$ & $42 / 78$ & 53.78 \\
\hline \multicolumn{14}{|c|}{ Average FT = fault tolerance } & 47.38 \\
\hline
\end{tabular}

In the proposed work, the QCA layout of the FTRA gate is converted into the corresponding hardware description language notations using the HDLQ Verilog library [18]. The HDLQ design tool, Verilog equivalent for QCA, consists of a Verilog HDL library of QCA devices, that is, MV, INV, FO, Crosswire $(\mathrm{CW})$, and L-shape wire with fault injection capability. The HDLQ-modeled design of the FTRA gate is shown in Figure 9, and an exhaustive testing of the HDLQ model of the FTRA gate is conducted with 32 input patterns in the presence of all possible single missing/additional cell defects. The design is simulated using the Verilog HDL simulator in the presence of faults to determine the corresponding outputs.

Testing of the FTRA gate generated 78 unique fault patterns at the output, as shown in Table 4. Due to huge volume of data, the fault pattern table is partially shown. In the fault patterns study shown in the Table $4, a_{i}$ is the five-bit pattern with an equivalent decimal value of $i$. For example, $a_{0}$ represents 00000 (decimal 0), and $a_{31}$ represents 11111 (decimal 31). In Table 4, first two columns present the correct behaviour of FTRA gate, that is, for a particular input vector corresponding expected output vector. Each row of the Table 4 represents the output generated after the fault injection of different 78 modules of the HDLQ model (Figure 9), for example, for given input $a_{0}$, after the fault injection of different modules, the generated fault pattern is $a_{0}, a_{16}, a_{12}, \ldots, a_{1}, a_{0}$. From those fault patterns, we observed that there are average $47.38 \%$ successful patterns that produce the correct output, even when there is a fault.

\section{Conclusions}

This work presents a novel architecture of fault-tolerant reversible adder (FTRA) gate. Experimental results establish the fact that the proposed FTRA achieved significant improvements in reversible circuits over the existing ones. A reversible arithmetic logic unit is synthesized based on the FTRA proposed. This is first attempt to synthesize a fault-tolerant full adder/subtractor using only single reversible logic block (FTRA) with optimal quantum cost to avoid wire-crossing bottleneck. Also, the application of this fault-tolerant logic in QCA nanotechnology gets an extra advantage in fault-tolerant computing with effective $47.38 \%$ fault-free output in the presence of all possible single missing/additional cell defects.

Though, the clocking structure beneath the QCA cell layer is also very important and nontrivial research issue.

\section{Acknowledgment}

The authors would like to thank Mr. Samik Some, UG student of CSE department, NIT Durgapur, for his valuable help in testing the design using HDLQ tool.

\section{References}

[1] R. Landauer, "Irreversibility and heat generation in the computing process," IBM Journal of Research and Development, vol. 5, no. 3, pp. 183-191, 1961.

[2] C. H. Bennett, "Logical reversibility of computation," IBM Journal of Research and Development, vol. 17, no. 6, pp. 525-532, 1973.

[3] C. S. Lent, P. D. Tougaw, W. Porod, and G. H. Bernstein, "Quantum cellular automata," Nanotechnology, vol. 4, no. 1, pp. 49-57, 1993.

[4] Z. Guan, W. Li, W. Ding, Y. Hang, and L. Ni, "An arithmetic logic unit design based on reversible logic gates," in Proceedings of the 13th IEEE Pacific Rim Conference on Communications, Computers and Signal Processing (PacRim '11), pp. 925-931, August 2011.

[5] M. Morrison and N. Ranganathan, "Design of a reversible ALU based on novel programmable reversible logic gate structures," in Proceedings of IEEE Computer Society Annual Symposium on VLSI (ISVLSI '11), pp. 126-131, Washington, DC, USA, July 2011.

[6] Y. Syamala and A. V. N. Tilak, "Reversible arithmetic logic unit," in Proceedings of the 3rd International Conference on Electronics Computer Technology (ICECT '11), pp. 207-211, April 2011.

[7] J. W. Bruce, M. A. Thornton, L. Shivakumaraiah, P. S. Kokate, and $\mathrm{X} . \mathrm{Li}$, "Efficient adder circuits based on a conservative reversible logic gate," in Proceedings of IEEE Symposium on VLSI, pp. 83-88, Washington, DC, USA, 2002.

[8] M. Haghparast and K. Navi, "A novel fault tolerant reversible gate for nanotechnology based systems," American Journal of Applied Sciences, vol. 5, no. 5, pp. 519-523, 2008. 
[9] Md. S. Islam, M. M. Rahman, Z. Begum, and M. Z. Hafiz, "Efficient approaches for designing fault tolerant reversible carry look-ahead and carry-skip adders," MASAUM Journal of Basic and Applied Sciences, vol. 1, no. 3, pp. 354-360, 2009.

[10] S. K. Mitra and A. R. Chowdhury, "Minimum cost fault tolerant adder circuits in reversible logic synthesis," in Proceedings of the 25th International Conference on VLSI Design (VLSID '12), pp. 334-339, January 2012.

[11] K. Walus, T. J. Dysart, G. A. Jullien, and R. A. Budiman, "QCADesigner: a rapid design and simulation tool for quantum-dot cellular automata," IEEE Transactions on Nanotechnology, vol. 3, no. 1, pp. 26-31, 2004.

[12] T. Toffoli, "Reversible computing," in Proceedings of the 7th Colloquium on Automata, Languages and Programming, pp. 632-644, Springer, London, UK, 1980.

[13] M. Perkowski, M. Lukac, P. Kerntopf et al., "A hierarchical approach to computer-aided design of quantum circuits," in Proceedings of the 6th International Symposium on Representations and Methodology of Future Computing Technology, pp. 201209, 2003.

[14] B. Parhami, "Fault-tolerant reversible circuits," in Proceedings of the 40th Asilomar Conference on Signals, Systems, and Computers (ACSSC '06), pp. 1726-1729, November 2006.

[15] X. Ma and F. Lombardi, "Fault tolerant schemes for QCA systems," in Proceedings of the 23rd IEEE International Symposium on Defect and Fault Tolerance in VLSI Systems (DFT '08), pp. 236-244, October 2008.

[16] M. B. Tahoori, J. Huang, M. Momenzadeh, and F. Lombardi, "Testing of quantum cellular automata," IEEE Transactions on Nanotechnology, vol. 3, no. 4, pp. 432-442, 2004.

[17] M. Momenzadeh, M. Ottavi, and F. Lombardi, "Modeling QCA defects at molecular-level in combinational circuits," in Proceedings of the 20th IEEE International Symposium on Defect and Fault Tolerance in VLSI Systems (DFT '05), pp. 208-216, October 2005.

[18] M. Ottavi, L. Schiano, F. Lombardi, and D. Tougaw, "HDLQ: a HDL environment for QCA design," Journal on Emerging Technologies in Computing Systems, vol. 2, no. 4, pp. 243-261, 2006. 

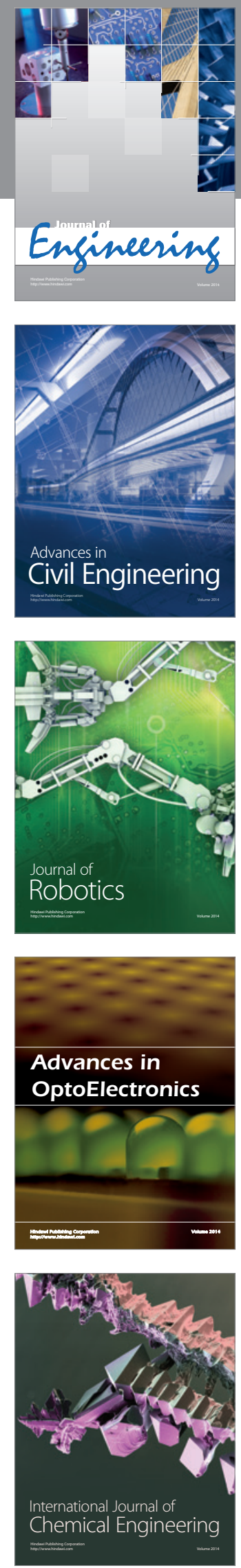

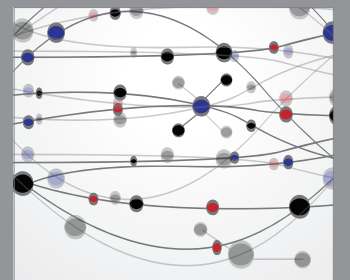

The Scientific World Journal
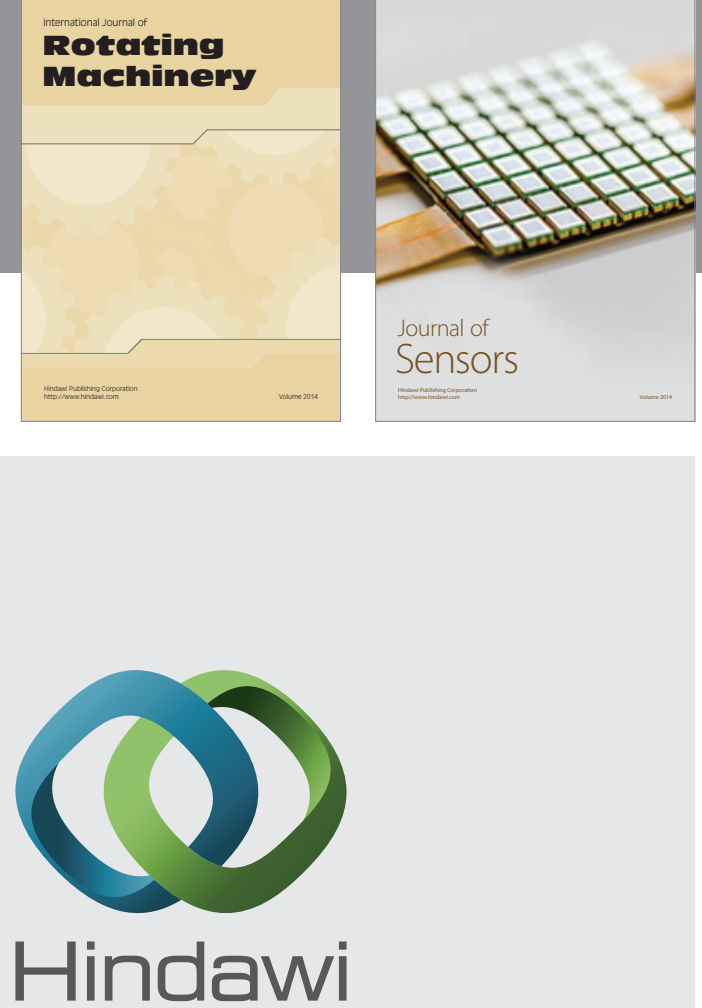

Submit your manuscripts at http://www.hindawi.com
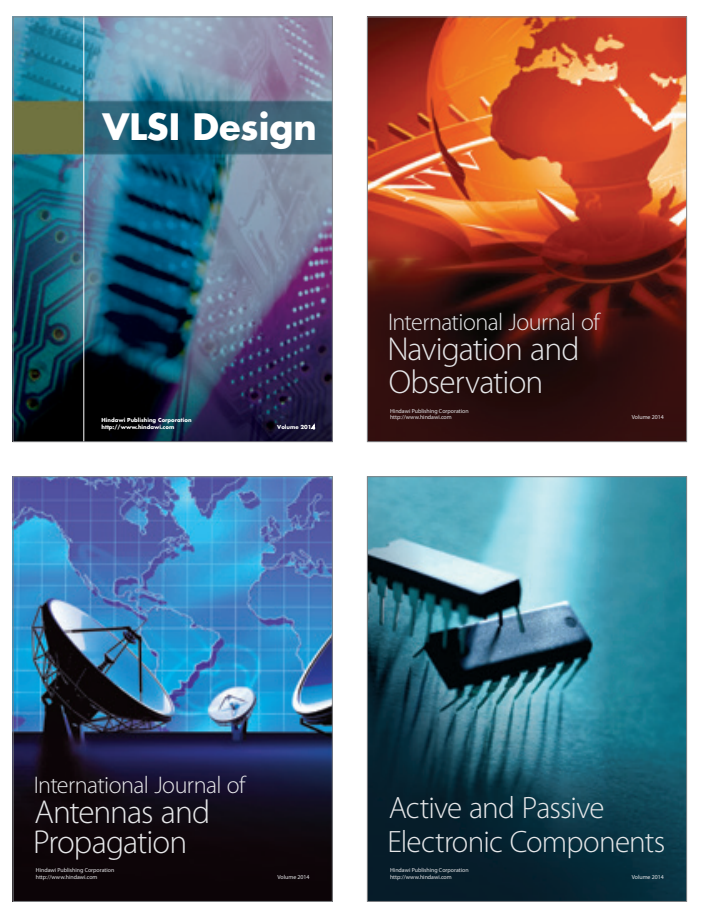
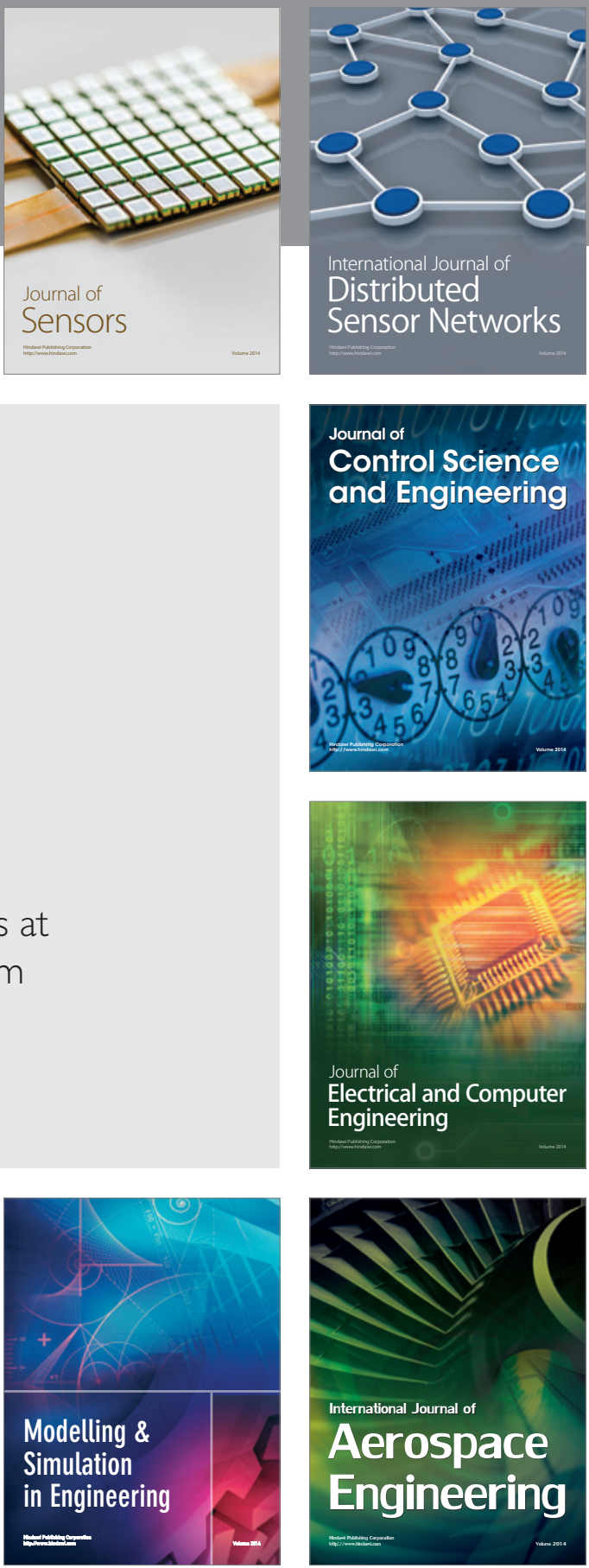

Journal of

Control Science

and Engineering
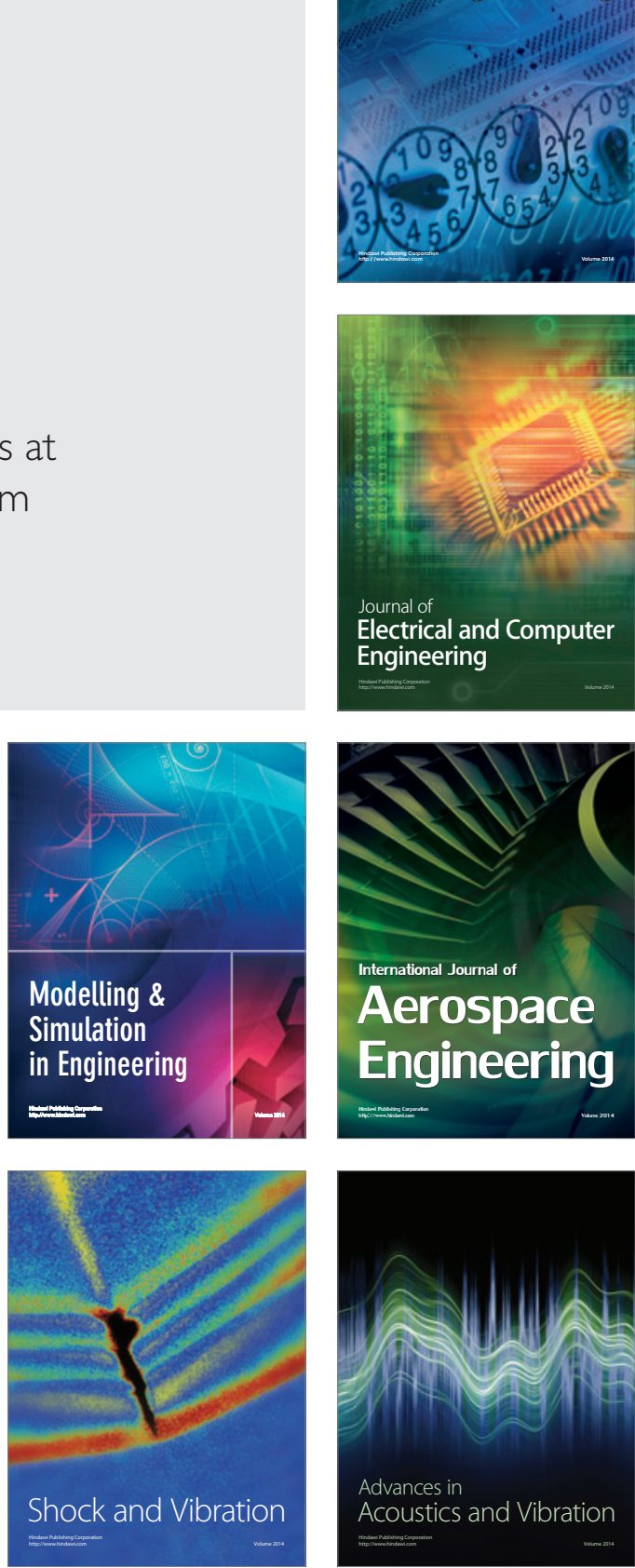